Pure and Applied Mathematics Quarterly

Volume 3, Number 3

(Special Issue: In honor of

Leon Simon, Part 2 of 2)

$773-783,2007$

\title{
Total Curvature of Graphs in Space
}

\author{
Robert Gulliver
}

\begin{abstract}
The Fáry-Milnor Theorem says that any embedding of the circle $S^{1}$ into $\mathbb{R}^{3}$ of total curvature less than $4 \pi$ is unknotted. More generally, a (finite) graph consists of a finite number of edges and vertices. Given a topological type of graphs $\Gamma$, what limitations on the isotopy class of $\Gamma$ are implied by a bound on total curvature? Especially: what does "total curvature" mean for a graph? I shall discuss several natural notions of the total curvature of a graph. Turning to the problem of isotopy type, I shall then focus on the notion of net total curvature $\mathcal{N}(\Gamma)$ of a graph $\Gamma \subset \mathbb{R}^{3}$, and outline the proof that if $\Gamma$ is homeomorphic to the $\theta$-graph, then $\mathcal{N}(\Gamma) \geq 3 \pi$; and if $\mathcal{N}(\Gamma)<4 \pi$, then $\Gamma$ is isotopic in $\mathbb{R}^{3}$ to a planar $\theta$-graph. Proofs will be given in full in [GY2].
\end{abstract}

\section{Introduction: TOtal CURVATURE}

The celebrated Fáry-Milnor theorem states that a curve in $\mathbb{R}^{n}$ of total curvature at most $4 \pi$ is unknotted [Fa], [Mi]. In the present paper, I shall emphasize the knotting dimension $n=3$. As a key step in his 1950 proof, John Milnor showed that for a smooth Jordan curve $\Gamma$ in $\mathbb{R}^{3}$, the total curvature equals one-half the integral over $e \in S^{2}$ of the number $\mu(e)$ of local maxima of the linear function $\langle e, \cdot\rangle$ along $\Gamma$ ([Mi], p. 252). This is equivalent to the definition of total curvature, as the supremum of total curvature of inscribed polygons, when $\Gamma$ is only continuous. The Fáry-Milnor theorem (even for $C^{0}$ curves) follows, since total curvature less than $4 \pi$ implies there is a unit vector $e_{0} \in S^{2}$ so that $\left\langle e_{0}, \cdot\right\rangle$ has a unique local maximum, and therefore that this linear function is increasing on one interval of $\Gamma$ and decreasing on the complementary interval. Without

Received January 12, 2006. 
changing the pointwise value of this height function, therefore, $\Gamma$ can be isotopically untwisted to a standard embedding of $S^{1}$ into $\mathbb{R}^{3}$. The Fenchel theorem ([Fen]), that any curve in $\mathbb{R}^{3}$ has total curvature at least $2 \pi$, also follows from Milnor's key step, since for all $e \in S^{2}$, the linear function $\langle e, \cdot\rangle$ assumes its maximum somewhere along $\Gamma$, implying $\mu(e) \geq 1$. Milnor's proof is independent from the earlier proof of Istvan Fáry, which takes a different approach $[\mathrm{Fa}]$.

I would like to discuss an extension of these results, replacing the simple closed curve by a finite graph $\Gamma$ in $\mathbb{R}^{3}$. $\Gamma$ consists of a finite number of points, the vertices, and a finite number of simple arcs, the edges, which each have as endpoints two of the vertices. The graphs I shall consider are assumed connected. The valence of a vertex $q$ is the number $\mathrm{d}(q)$ of edges which have $q$ as an endpoint. For convenience, I shall assume in this paper that each edge is of class $C^{2}$ up to its endpoints. I shall also assume that at each vertex $q$, the $\mathrm{d}(q)$ unit tangent vectors to the edges of $\Gamma$ are distinct.

The first difficulty one encounters in attempting to extend the results of Fáry and Milnor is a substantial one: to understand what is meant by the contribution to total curvature at a vertex of valence $\mathrm{d}(q) \geq 3$. Recall that, for a smooth closed curve $\Gamma$, the total curvature is

$$
\mathcal{C}(\Gamma)=\int_{\Gamma}|\vec{k}| d s
$$

where $s$ denotes arc length along $\Gamma$ and $\vec{k}$ is the curvature vector $\frac{d^{2} x}{d s^{2}}$, where $x(s) \in \mathbb{R}^{3}$ denotes the position in $\mathbb{R}^{3}$ at arc length $s$ along the curve. For a piecewise smooth curve, that is, a graph with vertices $q_{1}, \ldots, q_{N}$ having always valence $\mathrm{d}\left(q_{i}\right)=2$, the total curvature is readily generalized to

$$
\mathcal{C}(\Gamma)=\sum_{i=1}^{N} \mathrm{c}\left(q_{i}\right)+\int_{\Gamma_{\mathrm{reg}}}|\vec{k}| d s,
$$

where the integral is taken over the separate $C^{2}$ edges of $\Gamma$; and where $c\left(q_{i}\right) \in[0, \pi]$ is the exterior angle formed by the two edges of $\Gamma$ which meet at $q_{i}$. That is, $\cos \left(\mathrm{c}\left(q_{i}\right)\right)=$ $\left\langle\frac{d x}{d s}\left(q_{i}^{+}\right), \frac{d x}{d s}\left(q_{i}^{-}\right)\right\rangle$. The exterior angle $\mathrm{c}\left(q_{i}\right)$ is the correct contribution to total curvature, since any sequence of smooth curves converging to $\Gamma$ in $C^{0}$, with $C^{1}$ convergence on compact subsets of each open edge, includes a small arc near $q_{i}$ along which the tangent vector changes from approximately $\frac{d x}{d s}\left(q_{i}^{-}\right)$to approximately $\frac{d x}{d s}\left(q_{i}^{+}\right)$. The greatest lower bound of the contribution to total curvature along this disappearing arc equals $c\left(q_{i}\right)$.

However, the total curvature of a graph with vertices of valence $\mathrm{d}\left(q_{i}\right) \geq 3$, is rather ambiguous, as we shall see in the next section. 


\section{Definitions of Total Curvature for Graphs}

When we turn our attention to a graph $\Gamma \subset \mathbb{R}^{n}$, we notice that the above definition for valence $\mathrm{d}(q)=2$ does not generalize in any obvious way to higher valence. Whatever the definition of total curvature $\mathcal{C}(\Gamma)$, in order to correspond with our intuition, we might reasonably expect it to satisfy conditions such as the following:

(geom): $\mathcal{C}(\Gamma)$ is the total mass of a Borel measure $\kappa$ on $\Gamma$, where for any open set $U \subset \Gamma, \kappa(U)$ depends only on $U$ and not on $\Gamma \backslash U$, in an $\mathbb{O}(n)$-invariant and parameterization-invariant manner;

(curve): $\mathcal{C}(\Gamma)$ is consistent with equation (1) when all vertices of $\Gamma$ have valence two;

(scal): $\mathcal{C}$ is invariant under homotheties of $\mathbb{R}^{n}$; and

(lsc): $\mathcal{C}$ is lower semi-continuous, i.e. $\mathcal{C}(\Gamma) \leq \lim \sup \mathcal{C}\left(\Gamma_{k}\right)$ whenever $\Gamma_{k}$ is a sequence of graphs with homeomorphisms $\Gamma \rightarrow \Gamma_{k}$ converging uniformly to the identity.

It turns out that these four natural conditions allow a wide variety of notions of total curvature for graphs (see Theorem 1 below). In particular, we may consider the following three definitions:

(ve) In the calculus of variations, a natural notion of total curvature is the variational total curvature $\mathcal{V C}(\Gamma)$, which may be defined as the least upper bound of the first variation of length over variation vector fields having pointwise norm $\leq 1$ [AA]. As the specific choice for $\mathrm{c}(q)$ in equation (1), this means that one should use

$$
\operatorname{vc}(q):=\left\|\sum_{i=1}^{d} T_{i}\right\|,
$$

where $\left\{T_{1}, \ldots, T_{d}\right\}$ are the unit tangent vectors to $\Gamma$ at $q$, pointing into the $d=\mathrm{d}(q)$ edges with $q$ as endpoint (thanks to Bill Allard). Note that $\mathcal{V C}(\Gamma)$ satisfies (geom), (scal) and (lsc) but not (curve).

(mc) The notion of maximal total curvature $\mathcal{M C}(\Gamma)$ is defined with $\operatorname{mc}(q)$, which includes all $\frac{d(d-1)}{2}$ exterior angles at a vertex of valence $d$. That is,

$$
\operatorname{mc}(q):=\sum_{1 \leq i<j \leq d} \arccos \left\langle T_{i},-T_{j}\right\rangle .
$$


This time, $\mathcal{M C}(\Gamma)$ satisfies all four conditions defined above, although for some purposes it may be unreasonably large.

(tc) In my previous work with Sumio Yamada on the density of an area-minimizing rectifiable set $\Sigma$ spanning $\Gamma$, we found that it was very useful to apply the Gauss-Bonnet formula to the cone, with a point $p$ of $\Sigma$ as vertex, over an Euler circuit of the double of $\Gamma$ [GY1] (see also the next sections of this paper). The relevant notion of total curvature in that context is cone total curvature $\mathcal{C}_{\text {tot }}(\Gamma)$, defined using $\operatorname{tc}(q)$ as the choice for $\mathrm{c}(q)$ in equation (1):

$$
\operatorname{tc}(q):=\sup _{e \in S^{2}}\left\{\sum_{i=1}^{d}\left(\frac{\pi}{2}-\arccos \left\langle T_{i}, e\right\rangle\right)\right\} .
$$

Note that in the case $\mathrm{d}(q)=2$ of topological curves, the supremum above is assumed at vectors $e$ coplanar with the tangent vectors $T_{1}$ and $T_{2}$ to $\Gamma$, and lying in the smaller angle between them, so that $\operatorname{tc}(q)$ is then the exterior angle $\mathrm{c}(q)$ at $q$. Thus $\mathcal{C}_{\text {tot }}(\Gamma)$ satisfies condition (curve). In fact, conditions (geom), (scal) and (lsc) are satisfied as well. The main result of [GY1] is that $2 \pi$ times the density of $\Sigma$ at any of its points is at most equal to $\mathcal{C}_{\text {tot }}(\Gamma)$. The same result had been proved by Eckholm, White and Wienholtz for the case of a simple closed curve [EWW]. In this case, taking $\Sigma$ to be the branched immersion of the disk given by Douglas [D1] and Radó [R], it follows that if $\mathcal{C}_{\text {tot }}(\Gamma) \leq$ $4 \pi$, then $\Sigma$ is embedded and therefore $\Gamma$ is unknotted. That is, [EWW] provided an independent proof of the Fáry-Milnor theorem. Nonetheless, due in particular to the lack of knowledge of boundary regularity for area-minimizing rectifiable sets enclosing $\Gamma$, no conclusion about the isotopy classes of graphs follows from the results of [GY1].

To state matters another way, the results of [GY1] do not provide any conclusions about the "unknottedness" of a graph under conditions on $\mathcal{C}_{\text {tot }}(\Gamma)$. Moreover, there does not appear to be any such result in the literature, whether or not related to minimal surfaces, nor an appropriate definition of total curvature of a graph. Yamada and I will shed some light on this situation in [GY2]; the present paper is an outline of some of the results and their consequences. A number of mathematicians have largely succeeded in extending the purely topological theory of knots (which does not include total curvature), such as the theory of Reidemeister moves, to graphs; see for example [Ka] and [Y].

We now have three competing notions $\mathcal{V C}(\Gamma), \mathcal{M C}(\Gamma)$ and $\mathcal{C}_{\text {tot }}(\Gamma)$ for total curvature of a graph; a fourth notion, called net total curvature, will be introduced in the next 
section, and turns out to be much more closely related to the problem of isotopy of graphs.

As indicated above, the possible notions of total curvature satisfying the natural conditions mentioned are extremely general:

Theorem 1. For each integer $d \geq 2$, let $f_{d}:[0, \pi]^{d(d-1) / 2} \rightarrow[0, \infty)$ be any Lipschitz continuous, symmetric function, with Lipschitz norm at most 1 . Define the contribution to total curvature at a vertex $q$ of valence $d$, with unit tangent vectors $T_{1}, \ldots, T_{d}$ pointing into the edges of $\Gamma$ at $q$, as

$$
\operatorname{cc}(q)=f_{d}\left(d_{S^{n-1}}\left(T_{1}, T_{2}\right), d_{S^{n-1}}\left(T_{1}, T_{3}\right), \ldots, d_{S^{n-1}}\left(T_{d-1}, T_{d}\right)\right) .
$$

Then

$$
\mathcal{C C}(\Gamma):=\sum_{i=1}^{N} \operatorname{cc}\left(q_{i}\right)+\int_{\Gamma_{\text {reg }}}|\vec{k}| d s
$$

satisfies conditions (geom), (curve), (scal) and (lsc) above. If we define specifically $f_{2}(x):=\pi-x$, then $\mathcal{C C}(\Gamma)$ also satisfies condition (curve).

Proof. Condition (geom) follows readily from the definition of $\operatorname{cc}(q)$, since the spherical distance $d_{S^{n-1}}\left(T_{i}, T_{j}\right)$ is invariant under the group $\mathbb{O}(n)$ of isometries of $\mathbb{R}^{n}$. Observe that the integrand $|\vec{k}| d s$ is invariant under homotheties of $\mathbb{R}^{n}$; condition (scal) follows. Condition (curve) requires only the specific choice $f_{2}(x)=\pi-x$, so that $\operatorname{cc}(q)$ is the exterior angle $\mathrm{c}(q)$. It was observed in [Mi] that condition (lsc) implies condition (curve). The lower semi-continuity (lsc) will be shown in [GY2].

\section{Net Total Curvature of a Graph}

We next introduce the notion of net total curvature $\mathcal{N}(\Gamma)$, which is the appropriate definition for generalizing to graphs Milnor's approach to the total curvature of curves. For each unit tangent vector $T_{i}$ at a vertex $q, 1 \leq i \leq d=\mathrm{d}(q)$, let $\chi_{i}: S^{2} \rightarrow\{-1,+1\}$ be equal to -1 on the hemisphere with center at $T_{i}$, and +1 on the opposite hemisphere (values along the equator, which has measure zero, are arbitrary). We then define

$$
\operatorname{nc}(q):=\frac{1}{4} \int_{S^{2}}\left[\sum_{i=1}^{d} \chi_{i}(e)\right]^{+} d A_{S^{2}}(e) .
$$


In the case $\mathrm{d}(q)=2$, the integrand is positive (and equals 2 ) only on the set of unit vectors $e$ which have a negative inner product with both $T_{1}$ and $T_{2}$ (up to measure zero). This set is a lune bounded by semi-great circles orthogonal to $T_{1}$ and to $T_{2}$, and has spherical area equal to twice the exterior angle. So in this case, $\operatorname{nc}(q)$ is the exterior angle $\mathrm{c}(q)$, and $\mathcal{N}(\Gamma)$ satisfies the four conditions of section 2 above.

We would like to explain how the net total curvature $\mathcal{N}(\Gamma)$ of a graph is related to more familiar notions of total curvature. Recall that a graph $\Gamma$ has an Euler circuit (a closed path which traverses each edge of $\Gamma$ exactly once) if and only if its vertices all have even valence; this theorem of Euler is given an elementary proof in [O]. Of course, we do not have the hypothesis of even valence. We can attain that hypothesis for the double $\widetilde{\Gamma}$ of $\Gamma$ : the graph with the same vertices as $\Gamma$, but with two copies of each edge of $\Gamma$. Then at each vertex $q$, the valence as a vertex of $\widetilde{\Gamma}$ is $\widetilde{\mathrm{d}}(q)=2 \mathrm{~d}(q)$, which is even. By Euler's theorem, the double $\widetilde{\Gamma}$ of $\Gamma$ has an Euler circuit $\Gamma^{\prime}$, which may be thought of as a closed path which traverses each edge of $\Gamma$ exactly twice. Now at each of the points $\left\{q_{1}, \ldots, q_{d}\right\}$ of $\Gamma^{\prime}$ which are mapped to a vertex $q \in \Gamma$ of valence $d=\mathrm{d}(q)$, we may consider the exterior angle $\mathrm{c}\left(q_{i}\right)$. One-half the sum of these exterior angles, however, depends on the choice of the Euler circuit $\Gamma^{\prime}$. For example, if $\Gamma$ is the union of the $x$-axis and the $y$-axis in $\mathbb{R}^{3}$, then one might choose $\Gamma^{\prime}$ to have four right angles, or to have four straight angles, or two right and two straight, with completely different results. In order to form a version of total curvature at a vertex $q$ which depends only on the original graph $\Gamma$ and not on the choice of Euler circuit $\Gamma^{\prime}$, it is necessary to consider some exterior angles as partially balancing others. In the example just considered, where $\Gamma$ is the union of two orthogonal lines, opposite angles will balance each other completely, so that $\operatorname{nc}(q)=0$, regardless of the choice of Euler circuit of the double.

The nature of net total curvature is clearer when it is localized on $S^{2}$, analogously to [Mi]. In the case $\mathrm{d}(q)=2$, Milnor observed that the exterior angle at the vertex $q$ equals one-half the area of the set of $e \in S^{2}$ such that the linear function $\langle e, \cdot\rangle$, restricted to $\Gamma$, has a local maximum at $q$. In our case, we would like to describe $\operatorname{nc}(q)$ as one-half the integral over the sphere of the positive part of $\operatorname{nlm}(e, q)$, the number of net local maxima, which is the difference of the number of local maxima and the number of local minima at the points $\left\{q_{1}, \ldots, q_{d}\right\}$.

We need to show that

$$
\int_{S^{2}}[\operatorname{nlm}(e, q)]^{+} d A_{S^{2}}(e)
$$


is independent of the choice of Euler circuit, and in fact is equal to $2 \mathrm{nc}(q)$; this will follow from another way of computing $\operatorname{nlm}(e, q)$, in the next section (see Corollary 2 below).

\section{Some COMBinAtorics}

Definition 1. Let an Euler circuit $\Gamma^{\prime}$ of the double of $\Gamma$ be given. Then a vertex $q$ of $\Gamma$ corresponds to a number $\mathrm{d}(q)$ of vertices of $\Gamma^{\prime}$, where $\mathrm{d}(q)$ is the valence of $q$ as a vertex of $\Gamma$. If $q \in \Gamma$ is not a vertex, then we may consider $q$ as a vertex of valence $\mathrm{d}(q)=2$. Let $\operatorname{lmax}(e, q)$ be the number of local maxima of $\langle e, \cdot\rangle$ along $\Gamma^{\prime}$ at points over $q$, and similarly let $\operatorname{lmin}(e, q)$ be the number of local minima. Finally, define the number of net local maxima of $\langle e, \cdot\rangle$ at $q$ to be $\operatorname{nlm}(e, q)=\frac{1}{2}[\operatorname{lmax}(e, q)-\operatorname{lmin}(e, q)]$.

Remark 1. The definition of $\operatorname{nlm}(e, q)$ refers to a choice of the Euler circuit $\Gamma^{\prime}$ of the double of $\Gamma$; but $\Gamma^{\prime}$ is not unique, and indeed $\operatorname{lmax}(e, q)$ and $\operatorname{lmin}(e, q)$ may depend on the choice of $\Gamma^{\prime}$. However, we shall see in Corollary 1 below that the number $\operatorname{nlm}(e, q)$ of net local maxima is in fact independent of the choice of $\Gamma^{\prime}$.

I have included the factor $\frac{1}{2}$ in the definition of $\operatorname{nlm}(e, q)$ in order to agree with the difference of the numbers of local maxima and minima along $\Gamma$ itself, in those cases (such as $\mathrm{d}(q)=2$ ) where these numbers are unambiguously defined.

I shall assume for the rest of this section that a unit vector $e$ has been chosen, and that the linear function $\langle e, \cdot\rangle$ has only a finite number of critical points along $\Gamma$; this excludes $e$ from a subset of $S^{2}$ of measure zero. I shall also assume that that the graph $\Gamma$ is subdivided to include among the vertices all critical points of the linear function $\langle e, \cdot\rangle$, with of course valence $\mathrm{d}(q)=2$ if $q$ is an interior point of one of the original edges of $\Gamma$. For intuition, I think of the vector $e$ as pointing "up".

Definition 2. Choose a unit vector e. At a point $q \in \Gamma$ of valence $d=\mathrm{d}(q)$, let the upvalence $\mathrm{d}^{+}=\mathrm{d}^{+}(e, q)$ with respect to $e$ be the number of edges of $\Gamma$ with endpoint $q$ on which $\langle e, \cdot\rangle$ is greater than $\langle e, q\rangle$. Similarly, let the downvalence $\mathrm{d}^{-}(e, q)$ be the number of edges with endpoint $q$ along which $\langle e, \cdot\rangle$ is less than its value at $q$. Note that $\mathrm{d}(q)=\mathrm{d}^{+}(e, q)+\mathrm{d}^{-}(e, q)$.

Lemma 1. (Combinatorial Lemma) $\operatorname{nlm}(e, q)=\frac{1}{2}\left[\mathrm{~d}^{-}(e, q)-\mathrm{d}^{+}(e, q)\right]$.

The proof of this Lemma will be given in [GY2]. The reader may find it instructive to consider the example of a vertex $q \in \Gamma$ with $\mathrm{d}^{+}(e, q)=2$ and $\mathrm{d}^{-}(e, q)=3$, and various 
choices for the (local) Euler circuit $\Gamma^{\prime}$ of the double of $\Gamma$. Many of the 45 choices for $\Gamma^{\prime}$ will be seen to be equivalent in terms of local maxima and minima.

Corollary 1. The number of net local maxima $\operatorname{nlm}(e, q)$ is independent of the choice of Euler circuit $\Gamma^{\prime}$ of the double of $\Gamma$.

Proof. Given a direction $e \in S^{2}$, the upvalence and downvalence $\mathrm{d}^{ \pm}(e, q)$ at a vertex $q \in \Gamma$ are defined independently of the choice of $\Gamma^{\prime}$.

Corollary 2. $\operatorname{nc}(q)=\frac{1}{2} \int_{S^{2}}[\operatorname{nlm}(e, q)]^{+} d A_{S^{2}}$.

Proof. Consider $e \in S^{2}$. In the definition (3) of nc $(q), \chi_{i}(e)= \pm 1$ whenever $\pm\left\langle e, T_{i}\right\rangle<0$. But the number of $1 \leq i \leq d$ with $\pm\left\langle e, T_{i}\right\rangle<0$ equals $\mathrm{d}^{\mp}(e, q)$, so that

$$
\sum_{i=1}^{d} \chi_{i}(e)=\mathrm{d}^{-}(e, q)-\mathrm{d}^{+}(e, q)=2 \operatorname{nlm}(e, q)
$$

by Lemma 1.

Definition 3. For a graph $\Gamma \subset \mathbb{R}^{3}$ and $e \in S^{2}$, the multiplicity $\mu(e)=\mu_{\Gamma}(e):=$ $\sum\left\{\operatorname{nlm}^{+}(e, q): q\right.$ a vertex of $\left.\Gamma\right\}$.

Recall that we are including among the vertices all critical points of the linear function $\langle\cdot, e\rangle$ along $\Gamma$. Thus $\mu(e) \in \frac{1}{2} \mathbb{Z}$ may be described as half the number of net local maximum points along (any choice of) $\Gamma^{\prime}$.

\section{Theorem 2.}

$$
\mathcal{N}(\Gamma)=\frac{1}{2} \int_{S^{2}} \mu(e) d A_{S^{2}}(e) .
$$

The proof of Theorem 2 is related to [Mi], esp. p. 253, and will be presented in [GY2].

\section{NET TOTAL CURVATURE OF THE THETA GRAPH}

I shall discuss in this section a particularly simple homeomorphism type of graphs: there are only two vertices $q^{ \pm}$, and three edges $a_{1}, a_{2}, a_{3}$ with $\partial a_{i}=\left\{q^{+}, q^{-}\right\}, i=$ $1,2,3$. This is the graph of a lower-case theta in the normal printed form $\theta$, and is called the theta graph. The standard theta graph in $\mathbb{R}^{3}$ is the (isotopy class of) a planar circle plus one diameter. Note that not all theta graphs in $\mathbb{R}^{3}$ are isotopic: for example, $a_{1} \cup a_{2}$ might be knotted. 
For theta graphs, the notion of net total curvature allows us to draw conclusions analogous to the Fenchel theorem and the Fáry-Milnor theorem, which were proved for $\Gamma$ homeomorphic to $S^{1}$ :

Theorem 3. Suppose $\Gamma \subset \mathbb{R}^{3}$ is a theta graph. Then $\mathcal{N}(\Gamma) \geq 3 \pi$. If $\mathcal{N}(\Gamma)<4 \pi$, then $\Gamma$ is isotopic in $\mathbb{R}^{3}$ to the standard theta graph.

Note that $\mathcal{N}(\Gamma)<4 \pi$ does not imply $\mathcal{N}\left(\Gamma_{0}\right)<4 \pi$ for subgraphs $\Gamma_{0}$ of $\Gamma$. See [GY2] for an example.

Proof. We first show the Fenchel-type lower bound. Consider any $e \in S^{2}$ and the linear function $\langle\cdot, e\rangle$ along $\Gamma$. We need to show that $\mu(e) \geq 3 / 2$, from which Theorem 2 implies that $\mathcal{N}(\Gamma) \geq 3 \pi$.

If the maximum occurs at a vertex, say $q^{+}$, then $\operatorname{nlm}\left(e, q^{+}\right)=3 / 2$. Namely, at each of the three vertices $q_{1}^{+}, q_{2}^{+}, q_{3}^{+}$of $\Gamma^{\prime}$ corresponding to $q^{+},\langle\cdot, e\rangle$ has a (global hence local) maximum. It follows in this case that $\mu(e) \geq 3 / 2$.

Now suppose the maximum occurs at an interior point $q_{\max }$ of one of the original edges of $\Gamma$; then $\operatorname{nlm}\left(e, q_{\max }\right)=1$. Then for values of $t$ slightly smaller than $t_{\max }:=\max _{\Gamma}\langle\cdot, e\rangle$, there are exactly two points where $\langle\cdot, e\rangle$ takes the value $t$. As $t$ decreases toward $t_{\min }:=\min _{\Gamma}\langle\cdot, e\rangle$, the cardinality of the fibers of $\langle\cdot, e\rangle$ must change from two to at least three, since otherwise $\Gamma$ would be homeomorphic to $S^{1}$. If this cardinality increases at another critical point $q_{\text {crit }} \neq q^{ \pm}$, then $q_{\text {crit }}$ is a local maximum point and $\mu(e) \geq \operatorname{nlm}\left(e, q_{\max }\right)+\operatorname{nlm}\left(e, q_{\text {crit }}\right) \geq 2$, contrary to hypothesis. The remaining possibility is that the cardinality increases at a vertex, say $q^{+}$. Since the cardinality is increasing, we have the downvalence $\mathrm{d}^{-}\left(e, q^{+}\right)$strictly greater than the upvalence at $q^{+}$. By the Combinatorial Lemma $1, \operatorname{nlm}\left(e, q^{+}\right) \geq 1 / 2$, and in this case also, we have $\mu(e) \geq \operatorname{nlm}\left(e, q_{\max }\right)+\operatorname{nlm}\left(e, q^{+}\right) \geq 3 / 2$. This shows that for any theta graph $\Gamma$, and any $e \in S^{2}, \mu(e) \geq 3 / 2$, hence $\mathcal{N}(\Gamma) \geq 3 \pi$.

We next turn our attention to the Fáry-Milnor type upper bound, and assume $\mathcal{N}(\Gamma)<$ $4 \pi$. By Theorem 2, since $S^{2}$ has area $4 \pi$, it follows that there exists $e_{0} \in S^{2}$ with $\mu\left(e_{0}\right)<2$. Since $\mu\left(e_{0}\right)$ is a half-integer, we have $\mu\left(e_{0}\right)=3 / 2$ by the above discussion.

We shall show that there are at most two points $q \in \Gamma$, either vertices or critical points, with $\operatorname{nlm}\left(e_{0}, q\right)>0$. The linear function $\left\langle\cdot, e_{0}\right\rangle$ has a maximum at a point $q_{\max }$, with $\operatorname{nlm}\left(e_{0}, q_{\max }\right)=1$ or $3 / 2$ according as $d\left(q_{\max }\right)=2$ or 3 . If there is a second point with $\operatorname{nlm}\left(e_{0}, q\right)>0$, then $\operatorname{nlm}\left(e_{0}, q_{\max }\right)=1$ and $\operatorname{nlm}\left(e_{0}, q\right)=\frac{1}{2}$, which implies that $q$ is a vertex, say $q^{+}$, with $\mathrm{d}^{+}\left(q^{+}\right)=1, \mathrm{~d}^{-}\left(q^{+}\right)=2$ and $\mathrm{d}\left(q^{+}\right)=3$. 
Observe that for any $e \in S^{2}, \sum_{q} \operatorname{nlm}(e, q)=0$; in fact the number of local maxima along $\Gamma^{\prime}$ is equal to the number of local minima. It follows that $\mu\left(-e_{0}\right)=\sum\left[\operatorname{nlm}\left(e_{0}, q\right)\right]^{-}=$ $3 / 2$. We may apply the argument above, replacing $e_{0}$ with $-e_{0}$, to show that either $\operatorname{nlm}\left(e_{0}, q_{\text {min }}\right)=-3 / 2$ and $\operatorname{nlm}\left(e_{0}, q\right) \geq 0$ elsewhere; or $\operatorname{nlm}\left(e_{0}, q_{\text {min }}\right)=-1$ and and there is a second point, which must be a vertex, say $q^{-}$, with $\operatorname{nlm}\left(e_{0}, q^{-}\right)=-\frac{1}{2}$.

Thus, according to Lemma 1, there are four cases, depending on the numbers $\mathrm{d}\left(q_{\max }\right)$ and $\mathrm{d}\left(q_{\min }\right) \in\{2,3\}$, for the cardinality $\#\left(e_{0}, t\right)$ of the fiber $\left\{q \in \Gamma:\left\langle e_{0}, q\right\rangle=t\right\}$ as $t$ decreases from $t_{\max }$ to $t_{\min }$. Write $t^{ \pm}:=\left\langle q^{ \pm}, e_{0}\right\rangle$. The four cases, listed by $\left(\mathrm{d}\left(q_{\max }\right), \mathrm{d}\left(q_{\min }\right)\right)$, are:

$(3,3): \#\left(e_{0}, t\right) \equiv 3, t_{\min }<t<t_{\max }$.

$(3,2): \#\left(e_{0}, t\right)=3$ for $t^{-}<t<t_{\max } ; \#\left(e_{0}, t\right)=2$ for $t_{\min }<t<t^{-}$.

(2,3): $\#\left(e_{0}, t\right)=2$ for $t^{+}<t<t_{\max } ; \#\left(e_{0}, t\right)=3$ for $t_{\min }<t<t^{+}$.

$(2,2): \#\left(e_{0}, t\right)=2$ for $t^{+}<t<t_{\max }$ and for $t_{\min }<t<t^{-}$; \#(e $\left.e_{0}, t\right)=3$ for $t^{-}<t<t^{+}$.

In each of these four cases, we shall show that $\Gamma$ is isotopic in $\mathbb{R}^{3}$ to a planar graph with the same values of $\left\langle e_{0}, \cdot\right\rangle$. Let us consider the fourth case $(2,2)$ in detail, and observe that the other three cases follow in a similar fashion.

Write $P(t)$ for the plane of $\mathbb{R}^{3}$ defined by $\left\{x \in \mathbb{R}^{3}:\left\langle e_{0}, x\right\rangle=t\right\}$.

In the fourth case we have $\mathrm{d}\left(q_{\min }\right)=2=\mathrm{d}\left(q_{\max }\right)$, and there are distinct heights $t_{\min }<t^{-}<t^{+}<t_{\max }$ where $\#\left(e_{0}, t\right)$ changes value from 2 to 3 and back again to 2 .

For $t^{-}<t<t^{+}$, there are \# $\left(e_{0}, t\right)=3$ points in $\Gamma \cap P(t)$. One of the three edges of $\Gamma$, say $a_{2}$, lies entirely in the closed slab between $P\left(t_{-}\right)$and $P\left(t_{+}\right)$. As $t \rightarrow t^{ \pm}$, two of the points converge from a well-defined direction, since the three unit tangent vectors at $q^{ \pm}$are distinct. Letting $t$ decrease from $t=t^{+}$, there is an isotopy of the plane $P(t)$, varying continuously with $t$, so that the three points of $\Gamma \cap P(t)$ become collinear, with the edge $a_{2}$ meeting $P(t)$ at the middle point of the three collinear points. After a further isotopy in $\mathbb{R}^{3}$, we may achieve that $P(t) \cap \Gamma \subset Q$ for some plane $Q$ in $\mathbb{R}^{3}$ transverse to the planes $P(t)$.

For $t>t^{+}$and for $t<t^{-}$, we may continuously rotate and translate the planes $P(t)$ so that this top portion and this bottom portion of $\Gamma$ each lie inside $Q$. 
Now that we have shown $\Gamma$ is isotopic to a planar graph, we may find a further isotopy of the plane $Q$ which deforms $\Gamma$ into a circle with one chord. That is, $\Gamma$ is isotopic to the standard theta graph.

It should be noted that the same proof does not hold when $\Gamma$ has the homeomorphism type of a circle with two disjoint, parallel chords. Of course, the Fenchel-type lower bound for $\mathcal{N}(\Gamma)$ is larger than $3 \pi$, namely $4 \pi$. However, this graph may be embedded into $\mathbb{R}^{3}$ so that it is not isotopic to a planar graph, but so that a particular vector $e_{0}$ has the minimum value $\mu\left(e_{0}\right)=2$.

There remains a challenging question to determine the Fáry-Milnor type lower bound for the net total curvature of graphs in $\mathbb{R}^{3}$ of any specific homeomorphism type which are not isotopic to a standard embedding of that graph.

\section{REFERENCES}

[AA] W. Allard and F. Almgren, The structure of stationary one dimensional varifolds with positive density, Invent. Math 34 (1976), 83-97.

[D1] J. Douglas, Solution of the problem of Plateau, Trans. Amer. Math. Soc. 33(1931), 263-321.

[EWW] T. Ekholm, B. White, and D. Wienholtz, Embeddedness of minimal surfaces with total boundary curvature at most $4 \pi$, Annals of Mathematics 155 (2002), 109-234.

[Fa] M. I. Fáry, Sur la courbure totale d'une courbe gauche faisant un noeud, Bull. Soc. Math. France 77 (1949), 128-138.

[Fed] H. Federer, Geometric Measure Theory. Springer-Verlag, New York 1969.

[Fen] W. Fenchel, Über Krümmung und Windung geschlossener Raumkurven, Math. Ann. 101 (1929), 238-252.

[GY1] R. Gulliver and S. Yamada, Area density and regularity for soap film-like surfaces spanning graphs, Math. Z. 253 (2006), 315-331.

[GY2] R. Gulliver and S. Yamada, Total Curvature and isotopy of graphs in $\mathbb{R}^{3}$, in preparation.

[Ka] L. Kauffmann, Invariants of graphs in three-space, Trans. Amer. Math. Soc. 311 (1989), 697-710.

[Mi] J. Milnor, On the total curvature of knots, Annals of Math. 52 (1950), 248-257.

[O] Øystein Ore, Graphs and their Uses. Random House, New York 1963.

[R] T. Radó, On the Problem of Plateau. Springer, 1971.

[Y] S. Yamada, An invariant of spatial graphs, J. Graph Theory 13 (1989), 537-551.

\section{Robert Gulliver}

School of Mathematics

University of Minnesota

Minneapolis MN 55414

E-mail: gulliver@math.umn.edu 